\title{
Chitosan in Dermatology
}

\section{Zarrintaj $\mathrm{P}^{1 *}$ and Saeb $\mathrm{MR}^{2}$ \\ ${ }^{1}$ School of Chemical Engineering, College of Engineering, University of Tehran, Iran \\ 2Department of Resin and Additives, Institute for Color Science and Technology, Iran}

*Corresponding author: Payam Zarrintaj, School of Chemical Engineering, College of Engineering, University of Tehran, Tehran, Iran, Tel: +989143471735; Email: payam.zarrintaj@gmail.com

\section{Mini Review \\ Volume 3 Issue 3}

Received Date: October 03, 2018

Published Date: October 19, 2018

DOI: $10.23880 /$ cdoaj-16000154

\section{Abstract}

Benefiting from its intrinsic properties, chitosan resembles extracellular matrix (ECM), and, hence, various forms of chitosan have been practiced in dermatology. Nanofibers of chitosan have been successfully utilized in wound dressing for regeneration of various types of wounds. Chitosan nanoparticles have also been used in cell reprogramming, e.g. induced pluripotent stem cell, which is known as a new method for alopecia treatment, as signaled in drug delivery. Chitosan hydrogels are 3D macromolecular networks providing niche-like milieu that can enhance cellular activity. Thus, design and implementation of chitosan-based hydrogels enhances dermatological conditions. This mini-review highlights the importance and applications of chitosan-based structure as a harbinger for new emerging materials for dermatology uses.

Keywords: Chitosan; Dermatology; Nanofibers; Wound dressing; Wound regeneration

\section{Introduction}

Dermatology is a branch of medicine which deals with skin, nail, hair and the disease related to them. Tissue engineering is a practical route to facilitate treatment methods and contributes to enhanced regeneration of damaged tissue [1]. Appropriate biomaterial selection plays a key role in functional tissue repair [2]. Various materials have been practiced for dermatology via treating alopecia, wound and other diseases [3] Polysaccharides due to the structural and behavioral resemblance with extracellular matrix have attracted significant attention and appeared to a large degree reliable in mimicking tissue behavior $[4,5]$. Chitosan as a most favorable polysaccharide has been vastly used in dermatology form cosmetic to therapeutic uses. Thanks to antibacterial properties, chitosan becomes more widespread in skin care products, and according to statistics, dermatology has been benefited from various structures developed based on chitosan [6].

\section{Structural Forms of Chitosan Used in Dermatology}

\section{Nanofiber}

Nanostructures can enhance cellular activity so as to facilitate cell regeneration. Chitosan in the form of nanofiber can be produced via electrospinning. To achieve a proper fiber diameter and stability, chitosan should be blended with other polymers like poly (vinyl alcohol) [7]. Aligned nanofibers enhance the cell orientation and regeneration. Chitosan nanofibers have been widely 
utilized in wound dressing because of inherent antibacterial properties of chitosan [8].

\section{Particle}

Alopecia treatment and hair follicle regeneration requires an appropriate niche supplement. Chitosan particle due to the ECM-like behavior features proper 3D milieu for dermal papilla growth without disrupting the cell-cell interaction. Induced pluripotent stem cells (iPSCs) have been utilized as a new emerging method for regeneration. Chitosan nanoparticles have been utilized to reprogramming the cell to achieve the iPSCs, which can be applied in cell therapy [3].

\section{Hydrogel}

Hydrogel as a 3D network of polymer can retain water to provide with the tissues a suitable milieu. Chitosan hydrogel can be synthesized in different forms including thermosensitive and electroactive forms, achievable through various methods like grafting temperatureresponsive and conductive polymers. Thermosensitive chitosan can be applied non-invasively to appropriately cover targeted area. Moreover, hydrogel conductivity enhances the intracellular activities $[9,10]$. It has been reported that chitosan-based compounds can be used as nail lacquer to guard against dermatophyte infection that delivers drug to the nail as a topical agent [11].

\section{Conclusion}

Facile fabrication of chitosan compounds, its abundance, and along with its intrinsic properties gives reason to use it in dermatology applications. Chitosan can be prepared in various forms such as nanofiber, particle and hydrogel. Nanofibers can enhance cell proliferation and alignment in wound dressing to guarantee proper wound regeneration. Moreover, chitosan particles are widely utilized in drug delivery, cell reprogramming and niche formation exhibiting reliable outcomes. In addition, chitosan hydrogels accelerate the wound healing and provide 3D network for appropriate cellular activity. However, based on the final application of chitosan, a proper form of chitosan should be selected wisely.

\section{References}

1. Zarrintaj P, Moghaddam AS, Manouchehri S, Atoufi Z, Amiri A, et al. (2017) Can regenerative medicine and nanotechnology combine to heal wounds? The search for the ideal wound dressing. Nanomedicine 12(19): 2403-2422.

2. Zarrintaj P, Saeb MR, Ramakrishna S, Mozafari M (2018) Biomaterials Selection for Neuroprosthetics. Current Opinion in Biomedical Engineering 6: 99-109.

3. Nilforoushzadeh MA, Zare M, Zarrintaj P, Alizadeh E, Taghiabadi E, et al. (2018) Engineering the Niche for Hair Regeneration-A Critical Review. Nanomedicine: Nanotechnology, Biology and Medicine 15(1): 70-85.

4. Zarrintaj P, Manouchehri S, Ahmadi Z, Saeb MR, Urbanska AM, et al., (2018) Agarose-based biomaterials for tissue engineering. Carbohydr polym 187: 66-84.

5. Bakhshandeh B, Zarrintaj P, Oftadeh MO, Keramati F, Fouladiha H, et al. (2017) Tissue engineering; strategies, tissues, and biomaterials. Biotechnol Genet Eng Rev 33(2): 144-172.

6. Mohebbi S, Nezhad MN, Zarrintaj P, Jafari SH, Gholizadeh SS, et al. (2018) Chitosan in Biomedical Engineering: A Critical Review. Curr Stem Cell Res Ther 13: 1-1.

7. Shokrgozar MA, Mottaghitalab F, Mottaghitalab V, Farokhi M (2011) Fabrication of porous chitosan/poly (vinyl alcohol) reinforced single-walled carbon nanotube nanocomposites for neural tissue engineering. J Biomed Nanotechnol 7(2): 276-284.

8. Bhattarai N, Edmondson D, Veiseh O, Matsen FA, Zhang M (2005) Electrospun chitosan-based nanofibers and their cellular compatibility. Biomaterials 26(31): 6176-6184.

9. Zarrintaj P, Bakhshandeh B, Saeb MR, Sefat F, Rezaeian I, et al. (2018) Oligoaniline-based conductive biomaterials for tissue engineering, Acta biomater 72: 16-34.

10. Zarrintaj P, Ahmadi Z, Saeb MR, Mozafari M (2018) Poloxamer-based stimuli-responsive biomaterials. Materials Today: Proceedings 5(7): 15516-15523.

11. Ghannoum MA, Long L, Isham N, Bulgheroni A, Setaro M, et al. (2015) Ability of hydroxypropyl chitosan nail lacquer to protect against dermatophyte nail infection. Antimicrob Agents Chemother 59: 18441848. 